\title{
Analysis of soil-structure interaction in buildings with deep foundation
}

\section{Análises de interação solo-estrutura em edifícios com fundação profunda}

M. G. RITTER a

marioritter@unochapeco.edu.br https://orcid.org/0000-0002-4158-7325

M. L. MENEGOTTO mauromenegotto@gmail.com https://orcid.org/0000-0002-0660-9795

M. F. COSTELLA a, c costella@unochapeco.edu.br https://orcid.org/0000-0001-6471-9848

R. C. PAVAN

pavan.uffs@gmail.com https://orcid.org/0000-0001-6946-0839

S. E. PILZ a

silvio@unochapeco.edu.br https://orcid.org/0000-0003-0361-7658

\begin{abstract}
In this paper it is presented how the influence of soil-structure interaction (SSI) interferes on reinforced concrete structures in small buildings with deep foundations, with the objective of analyzing the influence of SSI on the loads and repressions, global stability and costs of materials. The analysis were based on numerical-computational simulations of a commercial building using CAD/TQS commercial software. The building was simulated with 4,6 and 8 floors with 3 different profiles of soils, generating 8 case studies. When considering SSI, the loads and repressions did not present significant variations and the parameters of global instability were within the normative recommendations. Among the variables analyzed, the material cost of the structure was the least affected item with the SSI consideration.
\end{abstract}

Keywords: drilled pile, reinforced concrete, group effect, global stability.

\section{Resumo}

Neste artigo é apresentado como a influência da interação solo-estrutura (ISE) interfere nas estruturas de concreto armado em edifícios de pequeno porte com fundações profundas, com o objetivo de analisar a influência da ISE nas cargas e recalques de pilares, estabilidade global e custos dos materiais. As análises basearam-se em simulações numérico-computacional de uma edificação comercial com o emprego do software comercial CAD/TQS. O edifício foi simulado com 4, 6 e 8 pavimentos com 3 perfis diferentes de solos, gerando 8 estudos de casos. Quando considerada a ISE, as cargas e recalques não apresentaram variações significativas e os parâmetros de instabilidade global ficaram dentro das recomendações normativas. Dentre as variáveis analisadas, o custo dos materiais da estrutura foi o item menos afetado com a consideração da ISE.

Palavras-chave: estacas escavadas, concreto armado, efeito de grupo, estabilidade global. 


\section{Introduction}

According to standard NBR 6122 of the Brazilian Association of Technical Standards [1], the soil-structure interaction is the structural analysis mechanism considering the deformability of foundations in conjunction with the superstructure.

According to Kausel [2], studies on Soil-Structure Interaction (SSI) developed gradually in the first decades of the first half of the twentieth century, evolving fast in the second half of the same century because of offshore and nuclear works and the emergence of studies using the finite element method and better performing computers to improve seismic safety.

In Brazil, SSI studies of surface foundations included the largest number of studies on the subject and were also the first to be conducted. Gusmão [3], Antoniazzi et al. [4] and Pavan et al. [5] developed SSI studies in foundations with spread footing foundations, Lima et al. [6] performed studies with mat foundations and Rosa et al. [7] with mixed foundations.

Studies involving deep foundations are fewer in number and were developed only in the last 15 years. This type of foundation has a greater complexity in numerical-computational simulations and was studied by Savaris et al. [8], Mota [9] and Borges [10].

Internationally, research on SSI has focused on dynamic aspects, as in Schepers [11], Restrepo and Jaramillo [12], Papadopoulos et al. [13] and Carbonari et al. [14].

According to Gusmão [15], SSI is not considered in most structural designs, i.e., the columns of buildings are treated as having unmovable supports. This fact was discovered in a national survey conducted with professionals who develop special, industrial and building designs, with only $3 \%$ of them taking SSI into account regularly. Venanzi, Salciarini and Tamagnini [16] also report that tall buildings are frequently designed without considering SSI in situations involving dynamic analysis.

In addition to being an interdisciplinary field, the main reasons for not considering SSI in designs involve the limited time available for computational simulations at the design level, in addition to the difficulty in performing numerical simulations. It is, therefore, important to develop research that demonstrates the behavior of structures of different sizes, with different types of foundations, when $\mathrm{SSI}$ is considered. This is reinforced by the increasing verticalization of the urban environment and construction systems, with these increasing spans and slenderness resulting in increasing loads on the foundations, making works more sensitive to settlements and deformations, directly influencing their performance.

The situation is similar for SSI studies involving dynamic analyses. So much so that according to Roberto and Sola [17] one can find a large number of specialists in soil dynamics who don't have an in-depth understanding of the dynamics of structures, while many specialists in structure dynamics don't know soil dynamics.

According to Iwamoto [18], Antoniazzi [19] and Mendes [20], the loads and settlements of the central columns tend to decrease while those of the periphery tend to increase when SSI is considered in the structural analysis of buildings. Studies by Jordão [21] and Delalibera [22] point to an increase in the global instability parameters when SSI is considered in structural designs. This previously-described behavior of loads, settlements and global stability represents the general trend or behavior in most studies on SSI.
For Crespo [23], the first floors of buildings function as a vierendeel beam formed by the spans between beams and columns, resulting in axial forces in the slabs and beams and with the other floors above behaving like a plate, suffering little influence of the movement of the floors below. As reported by Pavan, Costella and Guarnieri [5], the structural elements closest to the foundations have the greatest variations in their forces, regardless of the combination, and this is an important factor to be taken into account in the design of transition beams in basements.

According to Medeiros [24], the analytical solutions involving SSI available in the literature have very restricted applicability, making SSI a subject that requires further research. These SSI studies require the use of computer software for the modeling of structures. In Brazil, the most commonly used tool is the SISEs module, integrated with the commercial CAD/TQS software, which not only calculates the forces and settlements, but also sizes and designs structural elements.

Since SSI studies of small structures with deep foundations are scarce, the objective of this paper is to study the behavior of the infrastructure, superstructure and the variation of the material costs for foundations, beams, columns and slabs of a reinforced concrete building that takes SSI into account. The analyses were performed by varying the number of floors of the building, considering soil masses with different strength profiles and using deep foundations with drilled piles.

\section{Methodological procedures}

\subsection{Soil mass and admissible load analysis of the drilled piles}

For the characterization of the residual basalt clay soil, belonging to the Serra Geral geological formation in the region of the Paraná Basin, which covers a part of northern Rio Grande do Sul, more than half of Santa Catarina and the south and southwest of Paraná, 215 SPT (Standard Penetration Test) campaigns were studied, provided by a specialized company, totaling 816 holes. The objective of this collection was to qualify the soil of this region without a probabilistic or inferential aspect, obtaining three soil profiles for the design of piles with a small, medium and high load capacity. The geotechnical characteristics analyzed were the penetration resistance index (NSPT) at each meter, the depths of the water table and the bedrock. In this analysis, only probes that characterized the use of deep foundations were used, i.e., with several layers of clay soil, a common characteristic of the region under study, and with the bedrock at depths greater than $20 \mathrm{~m}$.

The adopted infrastructure was composed of pile caps and piles drilled by a mechanical auger. The Aoki and Velloso [25] method was used to determine the load capacity of the piles and the maximum compressive stress of the piles was set at values lower than $5 \mathrm{MPa}$, which was done to avoid the use of reinforcement along the piles' shaft. The usual reinforcement used at the top of the piles, for the transmission of loads and connection with the pile cap, was considered to be equal in all the performed analyses and as such, it was not considered as a cost comparison element. 
The maximum length of the piles was limited to $20 \mathrm{~m}$. The criterion for the definition of the pile diameter was established considering the most requested diameter in each pile cap in the ultimate limit state so that the characteristic acting load was at most $10 \%$ greater than the admissible load. This procedure was adopted to avoid the oversizing of the piles since the values of the diameters used were multiples of $10 \mathrm{~cm}$.

\subsection{Analysis of the superstructure and foundations}

The analyzed structure was an example of a building for commercial use, with a rectangular floor plan (Figure 1), with the analyses being carried out for 4, 6 and 8 floors. The C25 class of concrete was used for the columns, beams and slabs. The

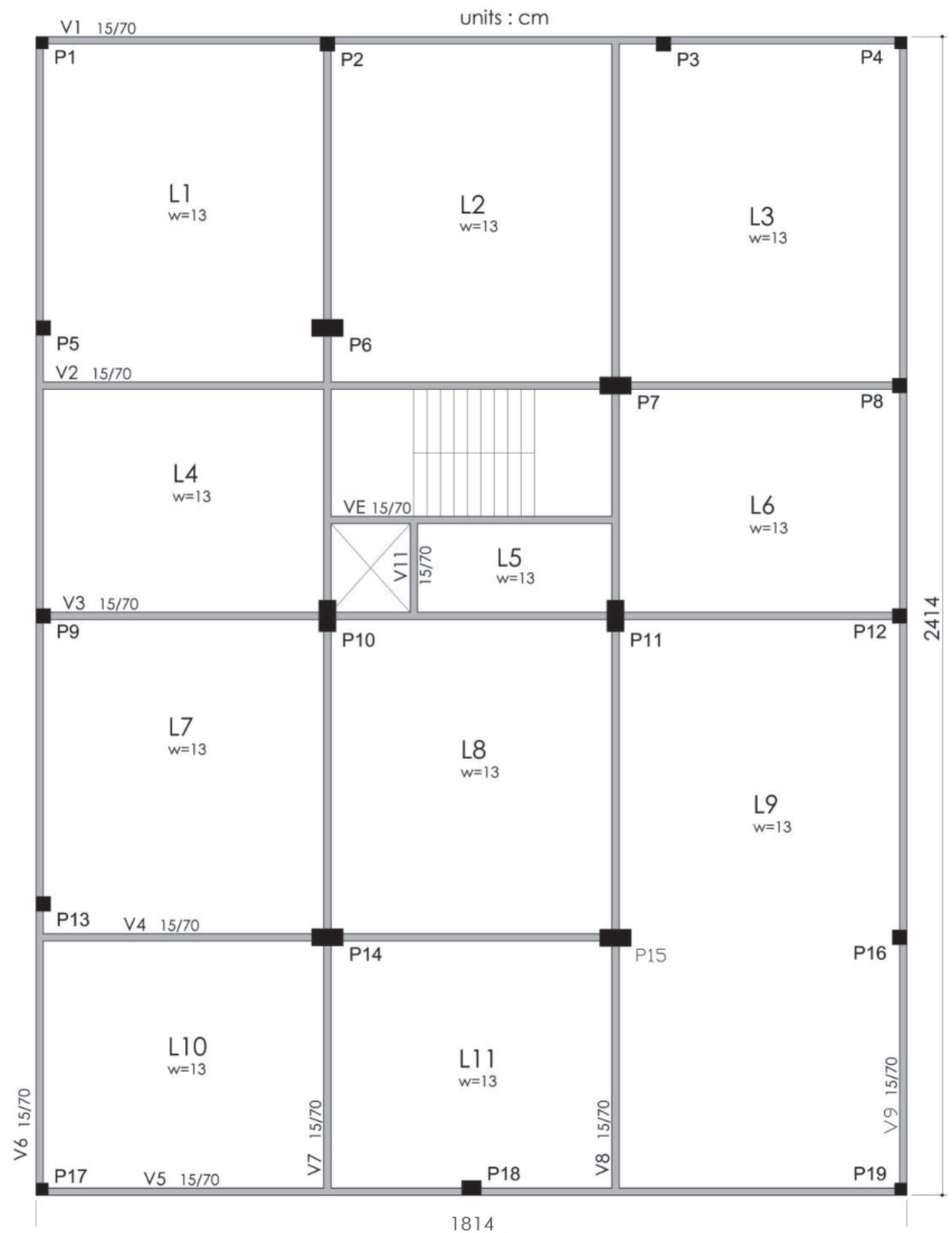

Figure 1

Commercial building with 4,6 and 8 floors 


\section{Table 1}

Dimension of the superstructure columns

\begin{tabular}{|c|c|c|c|}
\hline Column & $\begin{array}{l}\text { Dimensions of the columns } \\
\text { of the 4-floor buildings } \\
\text { (cm) }\end{array}$ & $\begin{array}{l}\text { Dimensions of the columns } \\
\text { of the 6-floor buildings } \\
(\mathrm{cm})\end{array}$ & $\begin{array}{l}\text { Dimensions of the columns } \\
\text { of the 8-floor buildings } \\
(\mathrm{cm})\end{array}$ \\
\hline 1 & $20 \times 20$ & $25 \times 25$ & $25 \times 25$ \\
\hline 2 & $30 \times 25$ & $30 \times 30$ & $30 \times 30$ \\
\hline 3 & $30 \times 25$ & $30 \times 30$ & $30 \times 30$ \\
\hline 4 & $20 \times 20$ & $25 \times 25$ & $25 \times 25$ \\
\hline 5 & $25 \times 25$ & $30 \times 25$ & $30 \times 30$ \\
\hline 6 & $45 \times 30$ & $55 \times 30$ & $65 \times 35$ \\
\hline 7 & $40 \times 30$ & $55 \times 30$ & $65 \times 35$ \\
\hline 8 & $25 \times 25$ & $30 \times 25$ & $30 \times 30$ \\
\hline 9 & $25 \times 25$ & $30 \times 25$ & $30 \times 30$ \\
\hline 10 & $30 \times 45$ & $30 \times 55$ & $35 \times 65$ \\
\hline 11 & $30 \times 45$ & $30 \times 55$ & $35 \times 65$ \\
\hline 12 & $25 \times 25$ & $30 \times 25$ & $30 \times 30$ \\
\hline 13 & $25 \times 25$ & $30 \times 25$ & $30 \times 30$ \\
\hline 14 & $45 \times 30$ & $55 \times 30$ & $65 \times 35$ \\
\hline 15 & $45 \times 30$ & $55 \times 30$ & $65 \times 35$ \\
\hline 16 & $25 \times 25$ & $30 \times 25$ & $30 \times 30$ \\
\hline 17 & $20 \times 20$ & $25 \times 25$ & $25 \times 25$ \\
\hline 18 & $30 \times 25$ & $40 \times 35$ & $40 \times 30$ \\
\hline 19 & $20 \times 20$ & $25 \times 25$ & $25 \times 25$ \\
\hline
\end{tabular}

C25 class of concrete was used for the pile caps and C20 for the piles.

Situations where there was interference between the pile caps or where the spacing between the axis of the piles was less than 3 times their diameter, were not considered in the analyses.

The columns were the only elements in the superstructure that had their dimensions modified depending on the number of floors. The dimensions of the columns in each of the three analyzed situations are shown in Table 1.

Since this was a commercial building, the considered accidental load was $0.30 \mathrm{kN} / \mathrm{m}^{2}$ and the lining load was $0.15 \mathrm{kN} / \mathrm{m}^{2}$. The parameters for considering the wind forces on the structure are shown in Table 2 .

The computational simulation consisted in the comparison of the structural models that had their columns supported on unmovable foundations (without SSI) with those columns supported on flexible foundations, both rotationally and translationally (with SSI). The CAD/TQS software with the SISEs module was used for the described procedure.

The beams and columns were simulated as bar elements, forming spatial frames. The slabs were simulated as grids consisting of bar elements. The rigid diaphragm effect represented by the slab in the spatial frame was considered in a simplified way, by increas-

\section{Table 2}

Parameters for wind loads

\begin{tabular}{ccccc}
\hline $\begin{array}{c}\text { Basic } \\
\text { speed } \\
\text { Vo }\end{array}$ & $\begin{array}{c}\text { Ground } \\
\text { factor } \\
\text { S1 }\end{array}$ & $\begin{array}{c}\text { Roughness } \\
\text { category } \\
\text { S2 }\end{array}$ & $\begin{array}{c}\text { Statistical } \\
\text { factor } \\
\text { S3 }\end{array}$ & $\begin{array}{c}\text { Building } \\
\text { class }\end{array}$ \\
\hline $45 \mathrm{~m} / \mathrm{s}$ & 1 & $\mathrm{IV}$ & 1 & $\mathrm{~B}$ \\
\hline
\end{tabular}

ing the lateral inertia of the beams. The pile caps were defined as rigid elements and the piles as bar elements connected to springs, which represented the soil mass.

The superstructure was designed with the simulation without SSI, with its supports being unmovable, from which the loads on the foundations were extracted in the ultimate limit state. After defining the loads on the foundations, the piles and pile caps were sized. The following steps were followed for the SSI simulation:

a) With the superstructure and its foundations defined, the reactions were obtained with and without wind for the structure calculated with its unmovable supports;

b) The settlements (displacements at the pile tip plus the elastic shortening of the pile shaft) were calculated with loads of the windless loading case, considering the group effects. The settlements in the pile tips were calculated with the Aoki and Lopes [26] method. The equivalent stiffnesses were calculated, dividing the forces (support reactions) applied by the respective settlements. The parameters used were the deformation modulus (Table 3 ) and the Poisson coefficient (Table 4);

\section{Table 3}

Soil deformation modulus estimates

\begin{tabular}{cc}
\hline Description of the soil type & $\begin{array}{c}\text { Typical values } \\
\left(\mathrm{KN} / \mathrm{m}^{2}\right)\end{array}$ \\
\hline Very soft clay (SPT $\leq 2)$ & 1000 \\
Soft clay (SPT 2 to 5) & 2000 \\
Medium clay (SPT 6 to 10) & 5000 \\
Stiff clay (SPT 11 to 19) & 8000 \\
Hard clay (SPT > 19) & 15000 \\
\hline
\end{tabular}




\section{Table 4}

Poisson coefficient estimates

\begin{tabular}{cc}
\hline Description of the soil type & $v$ \\
\hline Very soft clay (SPT $\leq$ 2) & 0.24 \\
Soft clay (SPT 2 to 5) & 0.23 \\
Medium clay (SPT 6 to 10) & 0.22 \\
Stiff clay (SPT 11 to 19) & 0.21 \\
Hard clay (SPT > 19) & 0.21 \\
\hline
\end{tabular}

Source: Adapted from TQS Informática [28]

c) The structure was resized with duly discretized piles until the base with Vertical Reaction Coefficients (VRCs) and Horizontal Reaction Coefficients (HRCs) through elastic bands, as shown in Figure 2.

In the extraction of the results of the performed analyses, the software automatically provided the loads on the foundations, the global stability parameters and the horizontal displacements at the top of the building. The settlements are not provided automatically for the cases without SSI. For the cases with SSI, the column settlements were read directly on the spatial frames after processing the integrated super $x$ infrastructure model.

In order to extract the settlements of the cases without SSI, the structures simulated with SSI were duplicated and then all the slabs, beams and columns of the structure were excluded. The following procedure consisted in manually adding only the loads for the case without SSI in the foundations that had all the piles and pile caps defined. In these models, only the piles and pile caps with the loads applied at their top were represented. The final procedure was the processing of this infrastructure and the reading of the settlements in the structural system formed by the piles and pile caps. It should be noted that the step described above is not part of the algorithm of the CAD/TQS software, because the unmovable supports do not have settlements.

\subsection{Material cost analysis}

The sizing, design and quantifying of the steel, concrete and formworks for the slabs, beams, columns and pile caps was performed

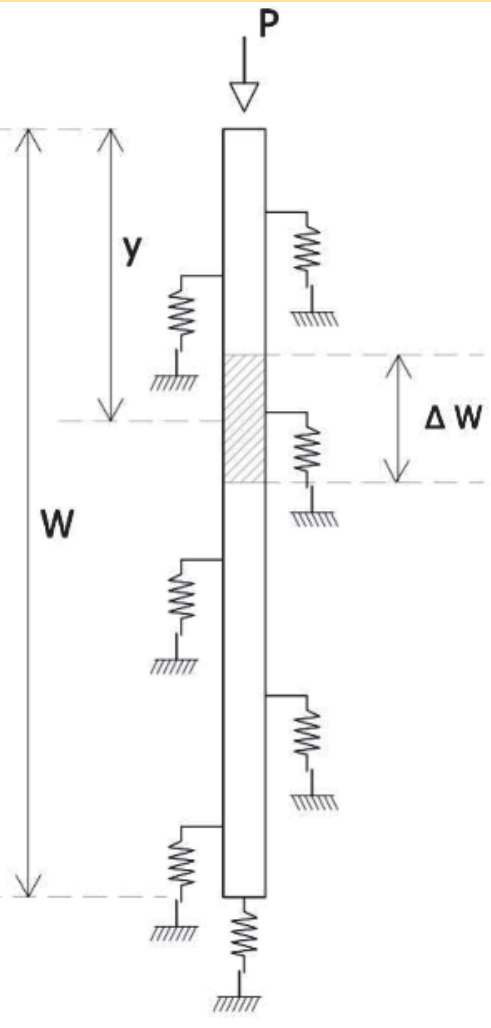

\section{Figure 2}

Representation of the discretized piles

Source: TQS Informática [28]

automatically by the CAD/TQS software. For the piles, these calculations were performed manually. Data from the National Research System of Civil Construction Costs and Indicators (SINAPI) were used for the cost composition of the concrete, formworks and steel. The costs were compared based on these data and considering the models with and without SSI, regarding the following aspects:

a) Concrete, formwork and steel costs;

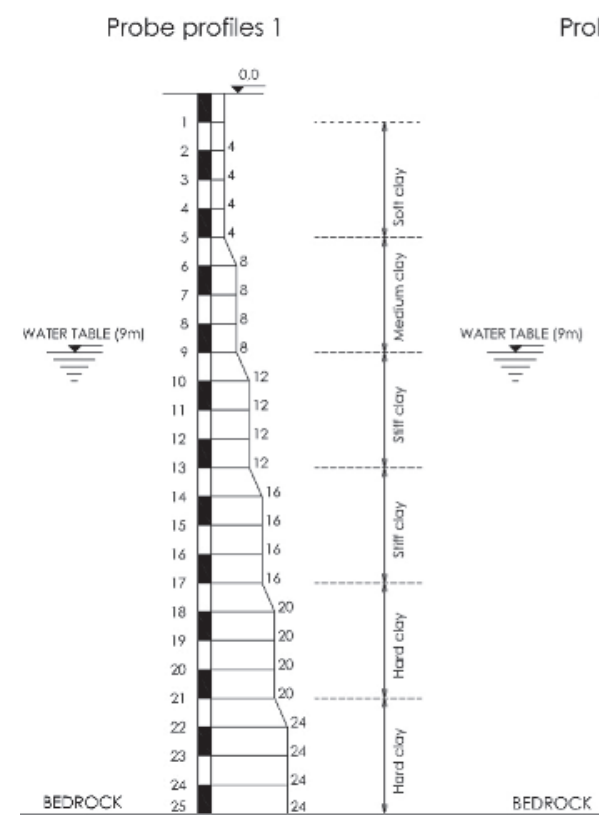

Probe profiles 2

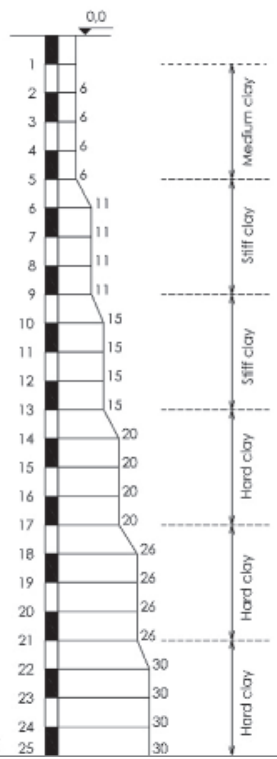

Probe profiles 3

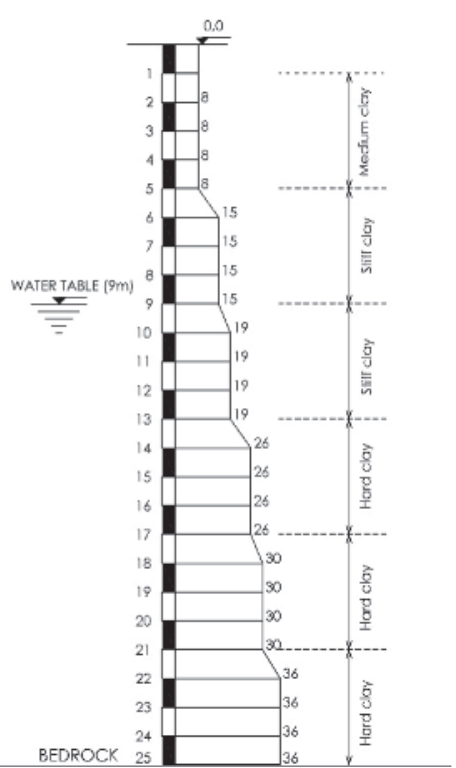

Figure 3

Geotechnical profiles 


\section{Table 5}

Load capacity according to the Aoki and Velloso method [25] for depths of $20 \mathrm{~m}$

\begin{tabular}{ccccccc}
\hline & Permissible load (kN) for 20 m deep piles & \multicolumn{3}{c}{ Permissible load variation (\%) } \\
\hline $\begin{array}{c}\text { Diameter } \\
(\mathbf{c m})\end{array}$ & $\begin{array}{c}\text { Probe profile 1 } \\
(+\mathbf{+ 1 0 \% )}\end{array}$ & $\begin{array}{c}\text { Probe profile 2 } \\
\mathbf{( + 1 0 \% )}\end{array}$ & $\begin{array}{c}\text { Probe profile 3 } \\
\mathbf{( + 1 0 \% )}\end{array}$ & $\begin{array}{c}\text { Profile 2 } \\
\text { to Profile 1 }\end{array}$ & $\begin{array}{c}\text { Profile 3 } \\
\text { to Profile 2 }\end{array}$ & $\begin{array}{c}\text { Profile 3 } \\
\text { to Profile 1 }\end{array}$ \\
\hline 30 & 279.95 & 363.88 & - & 23.07 & - & - \\
40 & 396.22 & 515.13 & 638.66 & 23.08 & 19.34 & 61.19 \\
50 & 524.15 & 681.34 & 841.50 & 23.07 & 19.03 & 60.55 \\
60 & 663.52 & 862.51 & 1061.61 & 23.07 & 18.75 & 60.00 \\
70 & 814.44 & 1058.75 & 1298.99 & 23.08 & 18.49 & 59.49 \\
80 & 976.80 & 1269.84 & 1553.75 & 23.08 & 18.27 & 59.07 \\
\hline \multicolumn{7}{c}{ Mean variation (\%) }
\end{tabular}

b) Structural element costs of the superstructure;

c) Structural element costs of the infrastructure;

d) The total cost of the superstructure and infrastructure;

e) Global cost (infrastructure plus superstructure).

\section{Results and discussion}

\subsection{Soil and admissible load analysis of the piles}

The mean geotechnical profiles obtained according to the methodology described above from the 215 probing campaigns are shown in Figure 3.

Table 5 shows the admissible loads of the piles as a function of their diameters and their values increased by $10 \%$, together with the percentage differences between the soils. The global safety factor used was equal to 2 .

\section{Table 6}

Data of the foundations of the 8-floor building

\begin{tabular}{cccc}
\hline \multicolumn{4}{c}{ Probe profile 2 } \\
\hline \multirow{2}{*}{ Column } & Block dimensions & Piles \\
\cline { 2 - 4 } & W $\mathbf{~ L ~ \times ~ H ~}$ & $\begin{array}{c}\boldsymbol{( c m}) \\
(\mathbf{c m})\end{array}$ & $\begin{array}{c}\text { Prof. } \\
(\mathrm{m})\end{array}$ \\
\hline P1 & $90 \times 90 \times 90$ & $1 \varnothing 60$ & 20 \\
P2 & $310 \times 100 \times 120$ & $2 \varnothing 70$ & 20 \\
P3 & $310 \times 100 \times 120$ & $2 \varnothing 70$ & 20 \\
P4 & $90 \times 90 \times 90$ & $1 \varnothing 60$ & 20 \\
P5 & $270 \times 90 \times 110$ & $2 \varnothing 60$ & 20 \\
P6 & $310 \times 310 \times 160$ & $4 \varnothing 70$ & 20 \\
P7 & $350 \times 350 \times 180$ & $4 \varnothing 80$ & 20 \\
P8 & $310 \times 100 \times 120$ & $2 \varnothing 70$ & 20 \\
P9 & $270 \times 90 \times 110$ & $2 \varnothing 60$ & 20 \\
P10 & $310 \times 310 \times 160$ & $4 \varnothing 70$ & 20 \\
P11 & $310 \times 310 \times 160$ & $4 \varnothing 70$ & 20 \\
P12 & $270 \times 90 \times 110$ & $2 \varnothing 60$ & 20 \\
P13 & $270 \times 90 \times 110$ & $2 \varnothing 60$ & 20 \\
P14 & $350 \times 350 \times 180$ & $4 \varnothing 80$ & 20 \\
P15 & $310 \times 310 \times 160$ & $4 \varnothing 70$ & 20 \\
P16 & $270 \times 90 \times 110$ & $2 \varnothing 60$ & 20 \\
P17 & $100 \times 100 \times 100$ & $1 \varnothing 70$ & 20 \\
P18 & $350 \times 110 \times 140$ & $2 \varnothing 80$ & 20 \\
P19 & $100 \times 100 \times 100$ & $1 \varnothing 70$ & 20 \\
\hline
\end{tabular}

For the pile with a diameter of $30 \mathrm{~cm}$ in probe profile 3 , with a depth of $20 \mathrm{~m}$, the compressive stress exceeded the value of 5 $\mathrm{MPa}$. As such, it was not used in the design of the foundations according to the adopted criterion. Instead, a $40-\mathrm{cm}$ diameter pile with a depth of $16 \mathrm{~m}$ was used, which had an admissible load of $459.80 \mathrm{kN}$.

\subsection{Analysis of the superstructure and foundations}

Table 6 shows the dimensions of the pile caps and the number of piles with their respective diameters and depths for the 8-floor building, determined with the loads applied to the foundations resulting from the analysis without SSI. When probe profile 1 was used for this number of floors, there was interference of the piles because their capacity was not high. This condition was therefore not considered, as explained in item 2.2.

\begin{tabular}{|c|c|c|c|}
\hline \multicolumn{4}{|c|}{ Probe profile 3} \\
\hline \multirow[b]{2}{*}{ Column } & \multirow{2}{*}{$\begin{array}{c}\text { Block dimensions } \\
\mathrm{W} \times \mathrm{L} \times \mathrm{H} \\
(\mathrm{cm})\end{array}$} & \multicolumn{2}{|c|}{ Piles } \\
\hline & & $\begin{array}{c}\varnothing \\
(\mathrm{cm})\end{array}$ & $\begin{array}{l}\text { Prof } \\
\text { (m) }\end{array}$ \\
\hline $\mathrm{P} 1$ & $80 \times 80 \times 80$ & $1 \varnothing 50$ & 20 \\
\hline P2 & $270 \times 90 \times 120$ & $2 \varnothing 60$ & 20 \\
\hline P3 & $270 \times 90 \times 120$ & $2 \varnothing 60$ & 20 \\
\hline P4 & $80 \times 80$ x 80 & $1 \varnothing 50$ & 20 \\
\hline P5 & $230 \times 80 \times 110$ & $2 \varnothing 50$ & 20 \\
\hline P6 & $270 \times 270 \times 150$ & $4 \varnothing 60$ & 20 \\
\hline P7 & $270 \times 270 \times 150$ & $4 \varnothing 60$ & 20 \\
\hline P8 & $270 \times 90 \times 120$ & $2 \varnothing 60$ & 20 \\
\hline P9 & $230 \times 80 \times 110$ & $2 \varnothing 50$ & 20 \\
\hline P10 & $270 \times 270 \times 150$ & $4 \varnothing 60$ & 20 \\
\hline P11 & $270 \times 270 \times 150$ & $4 \varnothing 60$ & 20 \\
\hline $\mathrm{P} 12$ & $230 \times 80 \times 110$ & $2 \varnothing 50$ & 20 \\
\hline $\mathrm{P} 13$ & $230 \times 80 \times 110$ & $2 \varnothing 50$ & 20 \\
\hline $\mathrm{P} 14$ & $310 \times 310 \times 160$ & $4 \varnothing 70$ & 20 \\
\hline P15 & $270 \times 270 \times 150$ & $4 \varnothing 60$ & 20 \\
\hline P16 & $230 \times 80 \times 110$ & $2 \varnothing 50$ & 20 \\
\hline $\mathrm{P} 17$ & $90 \times 90$ x 90 & $1 \varnothing 60$ & 20 \\
\hline P18 & $310 \times 100 \times 120$ & $2 \varnothing 70$ & 20 \\
\hline P19 & $90 \times 90 \times 90$ & $1 \varnothing 60$ & 20 \\
\hline
\end{tabular}


Table 7

Data of the foundations of the 6-floor building

\begin{tabular}{|c|c|c|c|}
\hline \multicolumn{4}{|c|}{ Probe profile 1} \\
\hline \multirow{2}{*}{ Column } & $\begin{array}{c}\text { Block } \\
\text { dimensions }\end{array}$ & \multicolumn{2}{|c|}{ Piles } \\
\hline & $\begin{array}{c}\text { W } \times \text { L } \times H \\
(\mathrm{~cm})\end{array}$ & $\begin{array}{c}\varnothing \\
(\mathrm{cm})\end{array}$ & $\begin{array}{l}\text { Prof. } \\
\text { (m) }\end{array}$ \\
\hline $\mathrm{Pl}$ & $90 \times 90 \times 90$ & $1 \varnothing 60$ & 20 \\
\hline P2 & $310 \times 100 \times 120$ & $2 \varnothing 70$ & 20 \\
\hline P3 & $310 \times 100 \times 120$ & $2 \varnothing 70$ & 20 \\
\hline P4 & 90 × 90 × 90 & $1 \varnothing 60$ & 20 \\
\hline P5 & $270 \times 90 \times 105$ & $2 \varnothing 60$ & 20 \\
\hline P6 & $310 \times 310 \times 165$ & $4 \varnothing 70$ & 20 \\
\hline P7 & $350 \times 350 \times 185$ & $4 \varnothing 80$ & 20 \\
\hline P8 & $270 \times 90 \times 105$ & $2 \varnothing 60$ & 20 \\
\hline PQ & $270 \times 90 \times 105$ & $2 \varnothing 60$ & 20 \\
\hline P10 & $310 \times 310 \times 165$ & $4 \varnothing 70$ & 20 \\
\hline P11 & $270 \times 270 \times 145$ & $4 \varnothing 60$ & 20 \\
\hline $\mathrm{P} 12$ & $270 \times 90 \times 105$ & $2 \varnothing 60$ & 20 \\
\hline $\mathrm{P} 13$ & $270 \times 90 \times 105$ & $2 \varnothing 60$ & 20 \\
\hline $\mathrm{P} 14$ & $350 \times 350 \times 185$ & $4 \varnothing 80$ & 20 \\
\hline P15 & $310 \times 310 \times 165$ & $4 \varnothing 70$ & 20 \\
\hline P16 & $270 \times 90 \times 105$ & $2 \varnothing 60$ & 20 \\
\hline $\mathrm{P} 17$ & $100 \times 100 \times 100$ & $1 \varnothing 70$ & 20 \\
\hline $\mathrm{P} 18$ & $350 \times 110 \times 140$ & $2 \varnothing 80$ & 20 \\
\hline P19 & $100 \times 100 \times 100$ & $1 \varnothing 70$ & 20 \\
\hline
\end{tabular}

\begin{tabular}{cccc}
\hline \multicolumn{4}{c}{ Probe profile 2 } \\
\hline & $\begin{array}{c}\text { Block } \\
\text { dimensions }\end{array}$ & \multicolumn{2}{c}{ Piles } \\
\cline { 2 - 5 } Column & W $\mathbf{L}$ L H & $\varnothing$ & Prof. \\
& $(\mathbf{c m})$ & $(\mathbf{c m})$ & $(\mathrm{m})$ \\
\hline P1 & $80 \times 80 \times 80$ & $\varnothing 50$ & 20 \\
P2 & $270 \times 90 \times 110$ & $2 \varnothing 60$ & 20 \\
P3 & $270 \times 90 \times 110$ & $2 \varnothing 60$ & 20 \\
P4 & $80 \times 80 \times 80$ & $1 \varnothing 50$ & 20 \\
P5 & $230 \times 80 \times 100$ & $2 \varnothing 50$ & 20 \\
P6 & $270 \times 270 \times 160$ & $4 \varnothing 60$ & 20 \\
P7 & $270 \times 270 \times 160$ & $4 \varnothing 60$ & 20 \\
P8 & $230 \times 80 \times 100$ & $2 \varnothing 50$ & 20 \\
P9 & $230 \times 80 \times 100$ & $2 \varnothing 50$ & 20 \\
P10 & $270 \times 270 \times 160$ & $4 \varnothing 60$ & 20 \\
P11 & $230 \times 230 \times 140$ & $4 \varnothing 50$ & 20 \\
P12 & $230 \times 80 \times 100$ & $2 \varnothing 50$ & 20 \\
P13 & $230 \times 80 \times 100$ & $2 \varnothing 50$ & 20 \\
P14 & $270 \times 270 \times 160$ & $4 \varnothing 60$ & 20 \\
P15 & $270 \times 270 \times 160$ & $4 \varnothing 60$ & 20 \\
P16 & $230 \times 80 \times 100$ & $2 \varnothing 50$ & 20 \\
P17 & $90 \times 90 \times 90$ & $1 \varnothing 60$ & 20 \\
P18 & $270 \times 90 \times 105$ & $2 \varnothing 60$ & 20 \\
P19 & $80 \times 80 \times 80$ & $1 \varnothing 50$ & 20 \\
\hline
\end{tabular}

\begin{tabular}{|c|c|c|c|}
\hline \multicolumn{4}{|c|}{ Probe profile 3} \\
\hline \multirow{2}{*}{ Column } & $\begin{array}{c}\text { Block } \\
\text { dimensions }\end{array}$ & \multicolumn{2}{|c|}{ Piles } \\
\hline & $\begin{array}{c}W \times L \times H \\
(\mathrm{~cm})\end{array}$ & $\begin{array}{c}\varnothing \\
(\mathrm{cm})\end{array}$ & $\begin{array}{l}\text { Prof. } \\
(\mathrm{m})\end{array}$ \\
\hline $\mathrm{Pl}$ & $70 \times 70 \times 70$ & $1 \varnothing 40$ & 20 \\
\hline P2 & $230 \times 80 \times 100$ & $2 \varnothing 50$ & 20 \\
\hline P3 & $230 \times 80 \times 100$ & $2 \varnothing 50$ & 20 \\
\hline P4 & $70 \times 70$ x 70 & $1 \varnothing 40$ & 20 \\
\hline P5 & $190 \times 70 \times 70$ & $2 \varnothing 40$ & 20 \\
\hline P6 & $230 \times 230 \times 155$ & $4 \varnothing 50$ & 20 \\
\hline P7 & $230 \times 230 \times 155$ & $4 \varnothing 50$ & 20 \\
\hline P8 & $230 \times 80 \times 100$ & $2 \varnothing 50$ & 20 \\
\hline P9 & $190 \times 70 \times 70$ & $2 \varnothing 40$ & 20 \\
\hline P10 & $230 \times 230 \times 155$ & $4 \varnothing 50$ & 20 \\
\hline $\mathrm{P} 11$ & $190 \times 190 \times 135$ & $4 \varnothing 40$ & 20 \\
\hline $\mathrm{P} 12$ & $190 \times 70 \times 70$ & $2 \varnothing 40$ & 20 \\
\hline $\mathrm{P} 13$ & $190 \times 70 \times 70$ & $2 \varnothing 40$ & 20 \\
\hline $\mathrm{P} 14$ & $230 \times 230 \times 155$ & $4 \varnothing 50$ & 20 \\
\hline P15 & $230 \times 230 \times 155$ & $4 \varnothing 50$ & 20 \\
\hline P16 & $190 \times 70$ x 70 & $2 \varnothing 40$ & 20 \\
\hline $\mathrm{P} 17$ & $80 \times 80 \times 80$ & $1 \varnothing 50$ & 20 \\
\hline P18 & $270 \times 90 \times 110$ & $2 \varnothing 60$ & 20 \\
\hline P19 & $80 \times 80$ × 80 & $1 \varnothing 50$ & 20 \\
\hline
\end{tabular}

Table 8

Data of the foundations of the 4-floor building

\begin{tabular}{|c|c|c|c|}
\hline \multicolumn{4}{|c|}{ Probe profile 1} \\
\hline \multirow{2}{*}{ Column } & $\begin{array}{c}\text { Block } \\
\text { dimensions }\end{array}$ & \multicolumn{2}{|c|}{ Piles } \\
\hline & $\begin{array}{c}W \times L \times H \\
(\mathrm{~cm})\end{array}$ & $\begin{array}{c}\varnothing \\
(\mathrm{cm})\end{array}$ & $\begin{array}{l}\text { Prof. } \\
\text { (m) }\end{array}$ \\
\hline $\mathrm{P} 1$ & $70 \times 70 \times 70$ & $1 \varnothing 40$ & 20 \\
\hline P2 & $230 \times 80 \times 100$ & $2 \varnothing 50$ & 20 \\
\hline P3 & $230 \times 80 \times 100$ & $2 \varnothing 50$ & 20 \\
\hline P4 & $70 \times 70 \times 70$ & $1 \varnothing 40$ & 20 \\
\hline P5 & $230 \times 80 \times 100$ & $2 \varnothing 50$ & 20 \\
\hline P6 & $350 \times 110 \times 140$ & $4 \varnothing 80$ & 20 \\
\hline P7 & $390 \times 120 \times 150$ & $4 \varnothing 90$ & 20 \\
\hline P8 & $230 \times 80 \times 100$ & $2 \varnothing 50$ & 20 \\
\hline PQ & $190 \times 70 \times 70$ & $2 \varnothing 40$ & 20 \\
\hline P10 & $110 \times 350 \times 140$ & $2 \varnothing 80$ & 20 \\
\hline $\mathrm{P} 11$ & $100 \times 340 \times 140$ & $2 \varnothing 80$ & 20 \\
\hline $\mathrm{P} 12$ & $190 \times 70 \times 70$ & $2 \varnothing 40$ & 20 \\
\hline $\mathrm{P} 13$ & $190 \times 70 \times 70$ & $2 \varnothing 40$ & 20 \\
\hline $\mathrm{P} 14$ & $390 \times 120 \times 150$ & $2 \varnothing 90$ & 20 \\
\hline P15 & $350 \times 110 \times 140$ & $2 \varnothing 80$ & 20 \\
\hline P16 & $190 \times 70 \times 70$ & $2 \varnothing 40$ & 20 \\
\hline P17 & $80 \times 80 \times 80$ & $1 \varnothing 50$ & 20 \\
\hline P18 & $270 \times 90 \times 110$ & $2 \varnothing 60$ & 20 \\
\hline P19 & $80 \times 80 \times 80$ & $1 \varnothing 50$ & 20 \\
\hline
\end{tabular}

\begin{tabular}{|c|c|c|c|}
\hline \multicolumn{4}{|c|}{ Probe profile 2} \\
\hline \multirow{2}{*}{ Column } & $\begin{array}{c}\text { Block } \\
\text { dimensions }\end{array}$ & \multicolumn{2}{|c|}{ Piles } \\
\hline & $\begin{array}{c}\mathrm{W} \times \mathrm{L} \times \mathrm{H} \\
(\mathrm{cm})\end{array}$ & $\begin{array}{c}\varnothing \\
(\mathrm{cm})\end{array}$ & $\begin{array}{l}\text { Prof. } \\
\text { (m) }\end{array}$ \\
\hline $\mathrm{Pl}$ & $60 \times 60 \times 60$ & $1 \varnothing 30$ & 20 \\
\hline P2 & $190 \times 70 \times 90$ & $2 \varnothing 40$ & 20 \\
\hline P3 & $190 \times 70 \times 90$ & $2 \varnothing 40$ & 20 \\
\hline P4 & $60 \times 60 \times 60$ & $1 \varnothing 30$ & 20 \\
\hline P5 & $190 \times 70 \times 70$ & $2 \varnothing 40$ & 20 \\
\hline P6 & $310 \times 100 \times 120$ & $2 \varnothing 70$ & 20 \\
\hline P7 & $310 \times 100 \times 120$ & $2 \varnothing 70$ & 20 \\
\hline P8 & $190 \times 70 \times 70$ & $2 \varnothing 40$ & 20 \\
\hline PQ & $190 \times 70 \times 70$ & $2 \varnothing 40$ & 20 \\
\hline P10 & $100 \times 310 \times 120$ & $2 \varnothing 70$ & 20 \\
\hline P11 & $90 \times 270 \times 100$ & $2 \varnothing 60$ & 20 \\
\hline $\mathrm{P} 12$ & $150 \times 60 \times 60$ & $2 \varnothing 30$ & 20 \\
\hline $\mathrm{P} 13$ & $190 \times 70 \times 70$ & $2 \varnothing 40$ & 20 \\
\hline P14 & $310 \times 100 \times 120$ & $2 \varnothing 70$ & 20 \\
\hline P15 & $310 \times 100 \times 120$ & $2 \varnothing 70$ & 20 \\
\hline $\mathrm{P} 16$ & $190 \times 70 \times 70$ & $2 \varnothing 40$ & 20 \\
\hline P17 & $70 \times 70 \times 70$ & $1 \varnothing 40$ & 20 \\
\hline P18 & $230 \times 80 \times 100$ & $2 \varnothing 50$ & 20 \\
\hline P19 & $70 \times 70 \times 70$ & $1 \varnothing 40$ & 20 \\
\hline
\end{tabular}

\begin{tabular}{|c|c|c|c|}
\hline \multicolumn{4}{|c|}{ Probe profile 3} \\
\hline \multirow{2}{*}{ Column } & $\begin{array}{c}\text { Block } \\
\text { dimensions }\end{array}$ & \multicolumn{2}{|c|}{ Piles } \\
\hline & $\begin{array}{l}W \times L \times H \\
(\mathrm{~cm})\end{array}$ & $\begin{array}{c}\varnothing \\
(\mathrm{cm})\end{array}$ & $\begin{array}{l}\text { Prof. } \\
\text { (m) }\end{array}$ \\
\hline $\mathrm{Pl}$ & $70 \times 70 \times 70$ & $1 \varnothing 40$ & 16 \\
\hline P2 & $190 \times 70 \times 70$ & $2 \varnothing 40$ & 16 \\
\hline P3 & $190 \times 70 \times 90$ & $2 \varnothing 40$ & 20 \\
\hline P4 & $70 \times 70 \times 70$ & $1 \varnothing 40$ & 16 \\
\hline P5 & $190 \times 70 \times 70$ & $2 \varnothing 40$ & 16 \\
\hline P6 & $270 \times 90 \times 110$ & $2 \varnothing 60$ & 20 \\
\hline P7 & $270 \times 90 \times 110$ & $2 \varnothing 60$ & 20 \\
\hline P8 & $190 \times 70 \times 70$ & $2 \varnothing 40$ & 16 \\
\hline PQ & $190 \times 70 \times 70$ & $2 \varnothing 40$ & 16 \\
\hline P10 & $90 \times 270 \times 110$ & $2 \varnothing 60$ & 20 \\
\hline $\mathrm{P} 11$ & $80 \times 230 \times 90$ & $2 \varnothing 50$ & 20 \\
\hline $\mathrm{P} 12$ & $190 \times 70 \times 70$ & $2 \varnothing 40$ & 16 \\
\hline $\mathrm{P} 13$ & $190 \times 70 \times 70$ & $2 \varnothing 40$ & 16 \\
\hline $\mathrm{P} 14$ & $270 \times 90 \times 110$ & $2 \varnothing 60$ & 20 \\
\hline P15 & $270 \times 90 \times 110$ & $2 \varnothing 60$ & 20 \\
\hline $\mathrm{P} 16$ & $190 \times 70 \times 70$ & $2 \varnothing 40$ & 16 \\
\hline $\mathrm{P} 17$ & $70 \times 70 \times 70$ & $1 \varnothing 40$ & 20 \\
\hline P18 & $190 \times 70 \times 90$ & $2 \varnothing 40$ & 20 \\
\hline P19 & $70 \times 70 \times 70$ & $1 \varnothing 40$ & 16 \\
\hline
\end{tabular}


Table 9

Summary of the behavior of the loads and settlements

\begin{tabular}{ccccc} 
& General trend & $\begin{array}{c}\text { Largest load variation } \\
(\%)\end{array}$ & $\begin{array}{c}\text { Largest settlement } \\
\text { without SSI } \\
\text { (mm) }\end{array}$ & $\begin{array}{c}\text { Largest settlement } \\
\text { with SSI } \\
\text { (mm) }\end{array}$ \\
\hline 8 floors - probe profile 2 & Yes & 6.49 & 22.10 & 21.48 \\
8 floors - probe profile 3 & Yes & 5.55 & 22.43 & 21.53 \\
6 floors - probe profile 1 & Yes & 5.31 & 17.16 & 16.83 \\
6 floors - probe profile 2 & Yes & 5.01 & 17.70 & 17.26 \\
6 floors - probe profile 3 & Yes & 7.07 & 18.97 & 18.40 \\
4 floors - probe profile 1 & No & 3.50 & 11.85 & 11.76 \\
4 floors - probe profile 2 & No & 2.95 & 11.95 & 11.85 \\
4 floors - probe profile 3 & No & 6.75 & 12.61 & 12.73 \\
\hline
\end{tabular}

Table 7 shows the dimensions of the pile caps, the number of piles and their respective depths for the 6-floor building, dimensioned with the loads applied to the foundations resulting from the analysis without SSI.

For the 4-floor building, the results of the sizing of the pile caps and piles are shown in Table 8.

Table 9 shows a summary of the behavior of the loads and settlements for the simulations with and without SSI. The general trend of loads and settlements reducing in the central columns while in- creasing in those of the periphery ceased to exist when the number of floors decreased. This phenomenon occurs for relatively low buildings because no vierendeel beams are formed on the first floors that function as bending beams. The explanation of this phenomenon is presented by Crespo [23], who describes the behavior of vierendeel beams in relation to the height of the building.

In general, the greatest variations in loads occurred in the buildings with the greatest number of floors and they had no significant changes, with the greatest variation being $7.07 \%$ according to
8 Floors / probe profile 2

Not considering the wind forces

Column

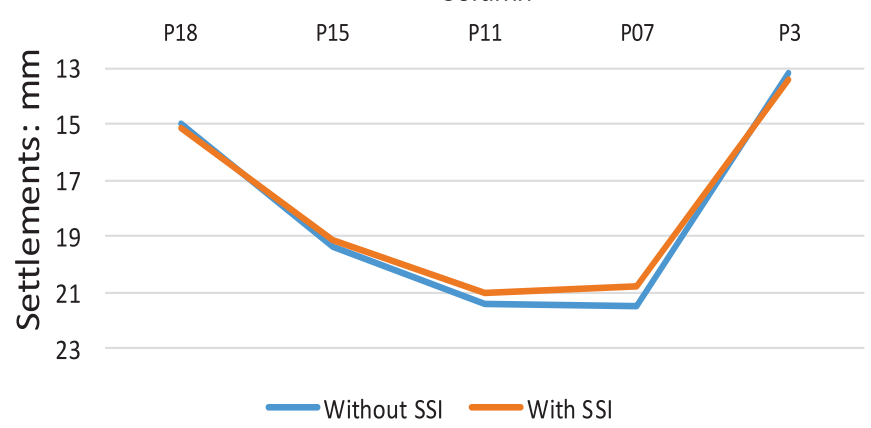

(a)

8 Floors / probe profile 3

Not considering the wind forces

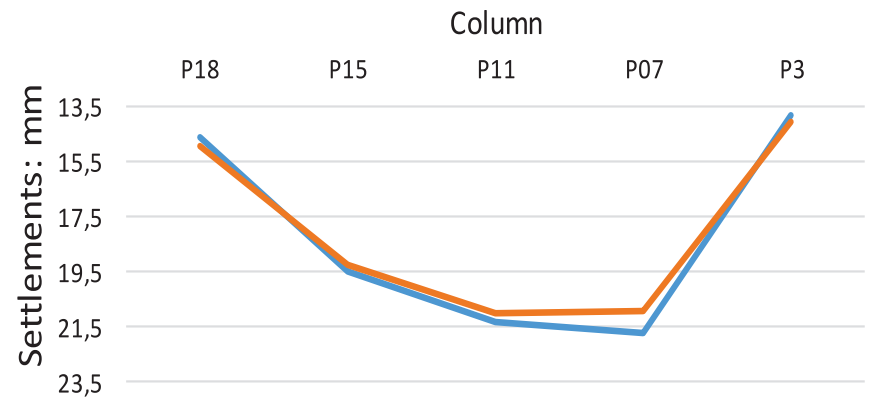

Without SSI —With SSI

(C)
8 Floors / probe profile 2

Considering the wind forces

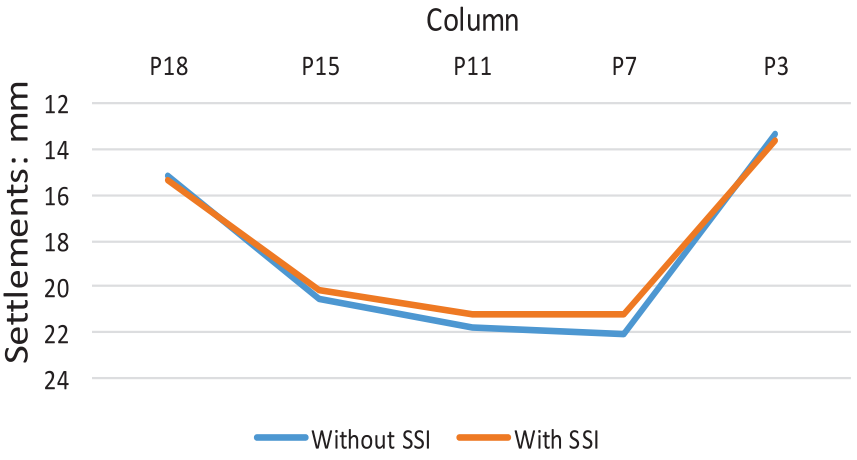

(b)

8 Floors / probe profile 3

Considering the wind forces

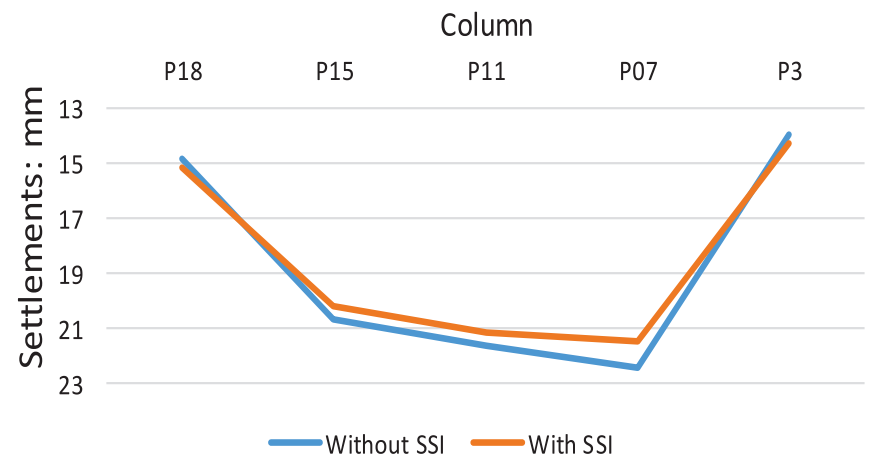

(d)

Figure 4

Settlement curve for the 8-floor building case 
Table 9. The variations described above were small because the buildings under analyses did not have transition beams with high stiffness. Another factor is that despite using 3 different geotechnical profiles, these did not have variations at the support points of the columns, causing the settlements to not have significant differences, thus reducing the effect of the SSI.

The settlement basin for the 8-floor building became smoother after considering the SSI, both for the case without wind and the case with wind, as can be seen in Figure 4, which shows the columns P18, P15, P11, P07 and P3 as examples. One can see that the differential settlements decreased after considering the SSI, in which the central columns had a decrease in settlements and those of the periphery had an increase.

The 6-floor building had similar behavior as the 8-floor building, as can be seen in Figure 5 for the columns P18, P15, P11, P07 and $\mathrm{P} 3$.
6 Floors / probe profile 1

Not considering the wind forces

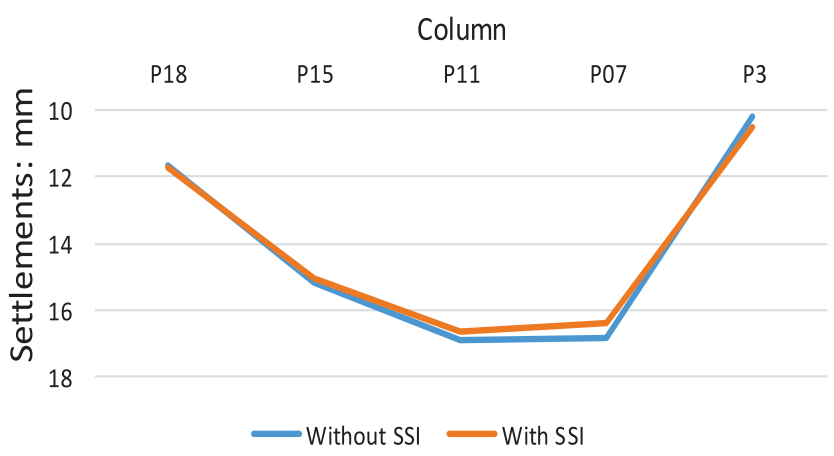

(a)

6 Floors / probe profile 2

Not considering the wind forces

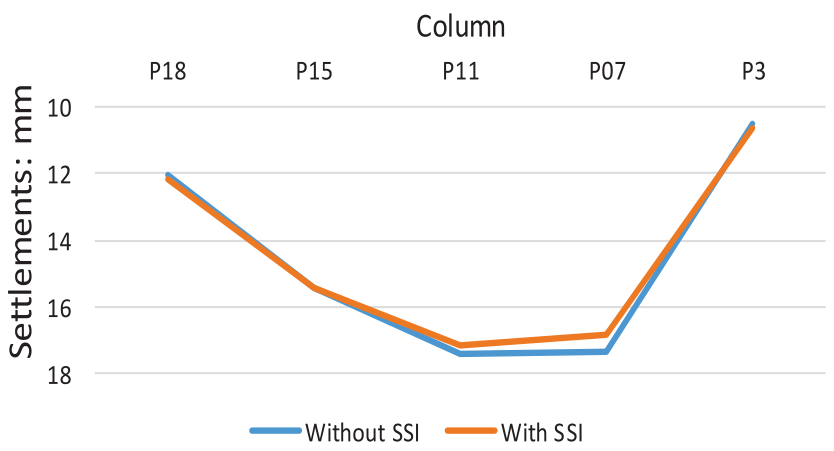

(c)

6 Floors / probe profile 3

Not considering the wind forces

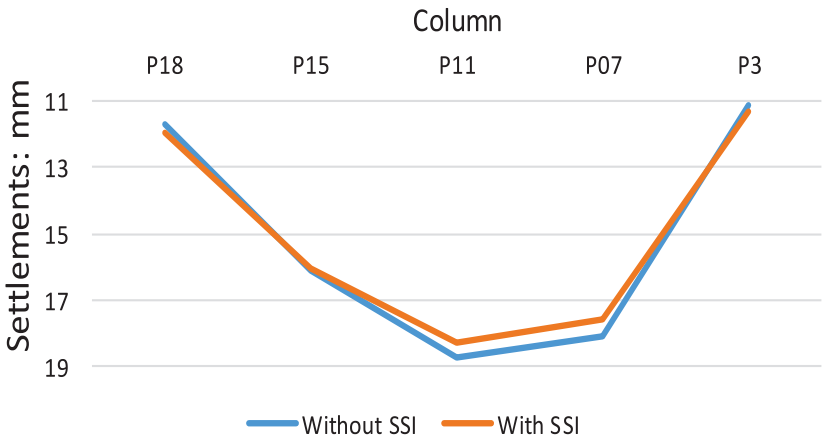

(e)
6 Floors / probe profile 1

Considering the wind forces

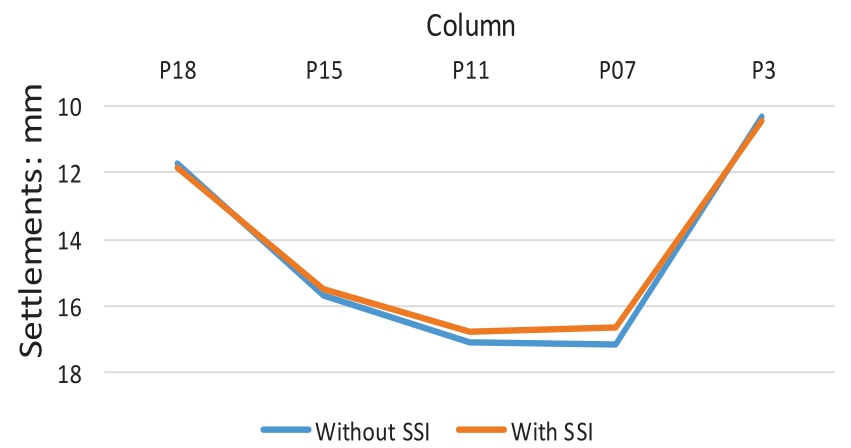

(b)

6 Floors / probe profile 2

Considering the wind forces

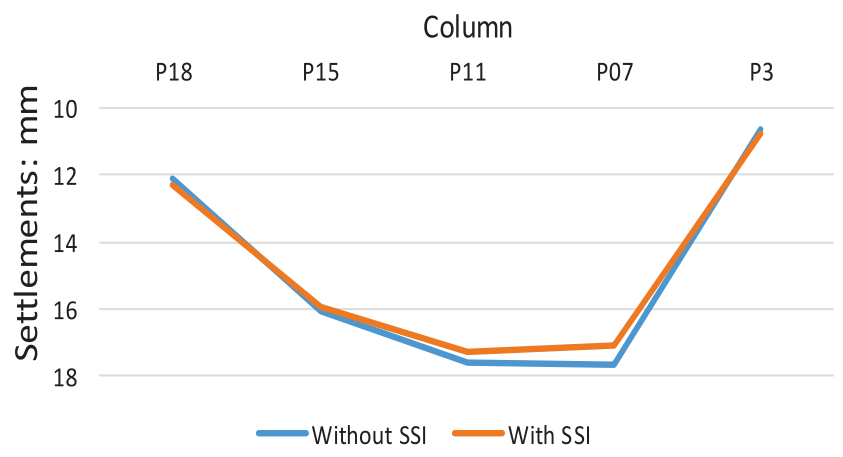

(d)

6 Floors / probe profile 3

Considering the wind forces

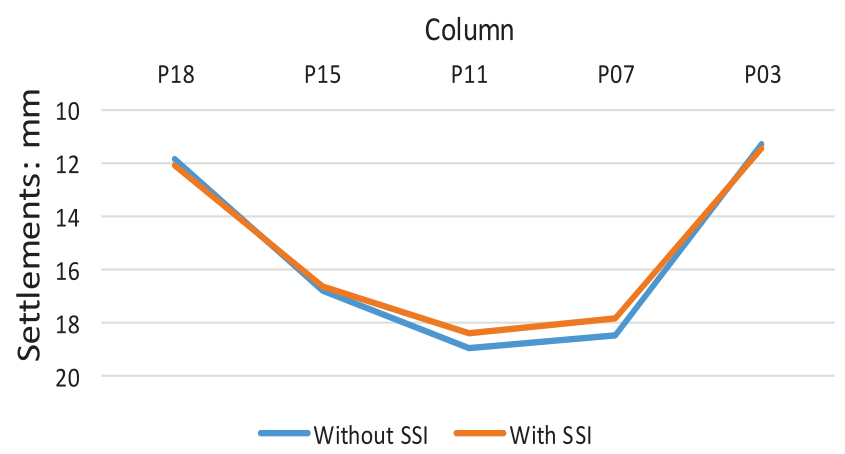

(f)

Figure 5

Settlement curve for the 6-floor building case 
Regarding the 4-floor building, the general trend ceased to exist, as can be seen in Figure 6 . For this building, the settlements had random behavior.

Regarding the instability parameter, the Gamma-Z coefficient always increased after considering the SSI. In general, the Gamma$\mathrm{Z}$ variation increased for more resistant soils and buildings with

4 Floors / probe profile 1

Not considering the wind forces

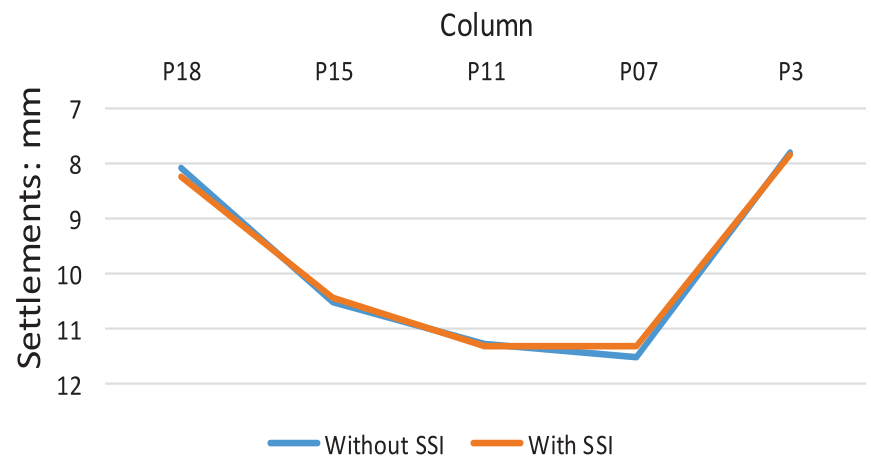

(a)

4 Floors / probe profile 2

Not considering the wind forces

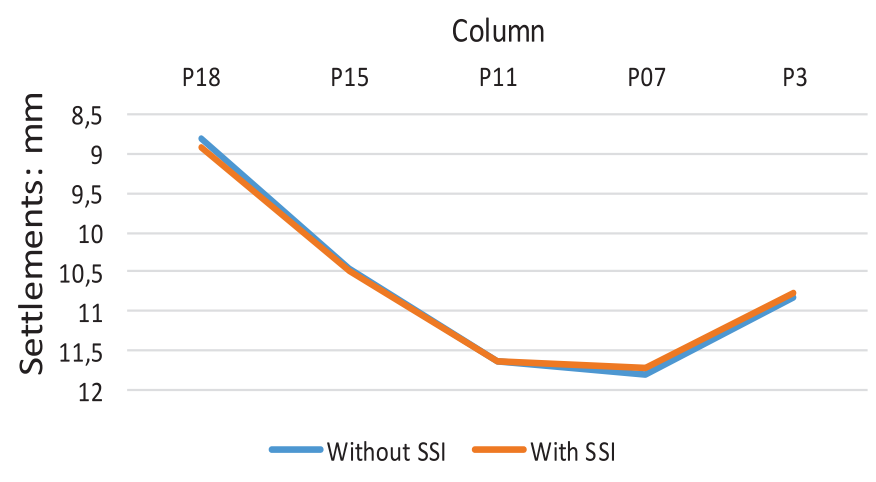

(C)

4 Floors / probe profile 3

Not considering the wind forces

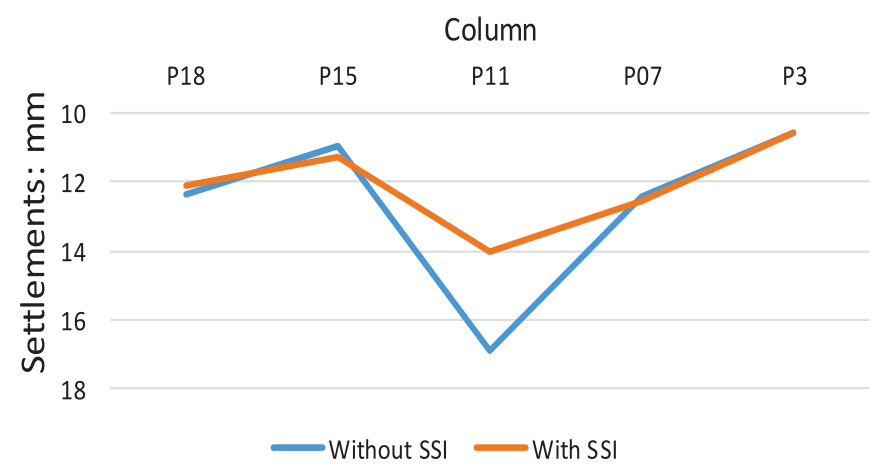

(e) fewer floors (Table 10). The largest variation found was $8.77 \%$. This occurs because when SSI is considered, the supports are no longer unmovable, thus increasing the horizontal displacements that directly influence the global Z-Gamma instability parameter. However, this instability fell within limits prescribed by the NBR 6118 standard.

4 Floors / probe profile 1

Considering the wind forces

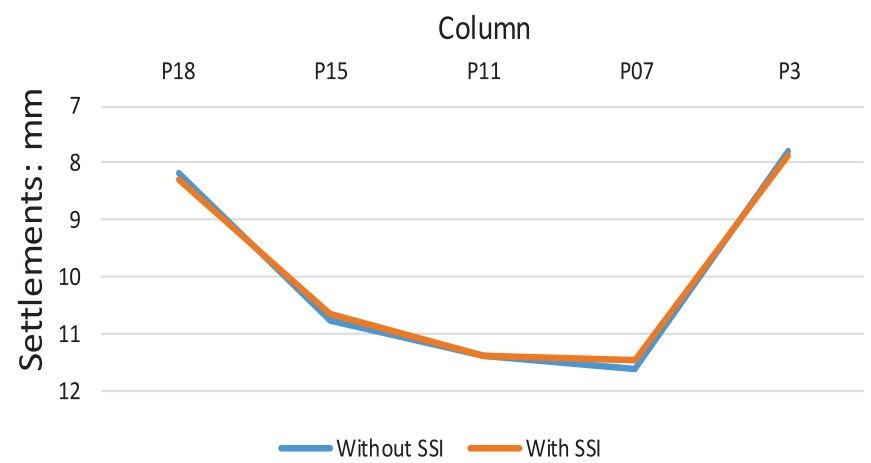

(b)

4 Floors / probe profile 2

Considering the wind forces

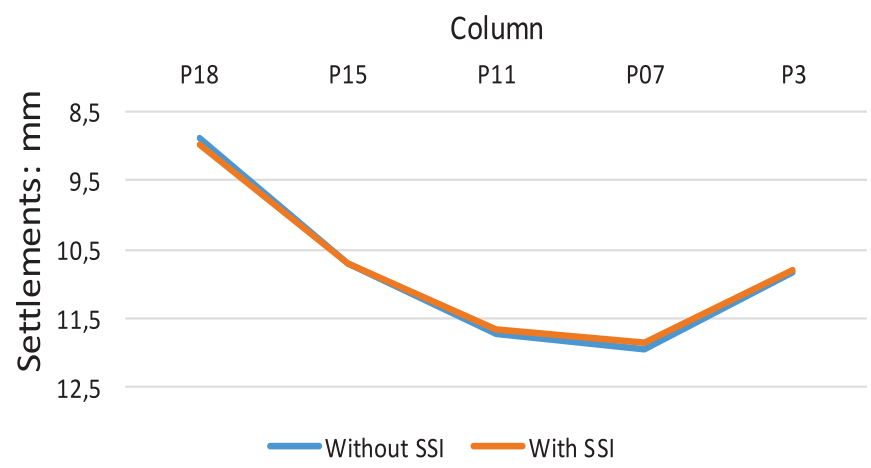

(d)

4 Floors / probe profile 3

Considering the wind forces

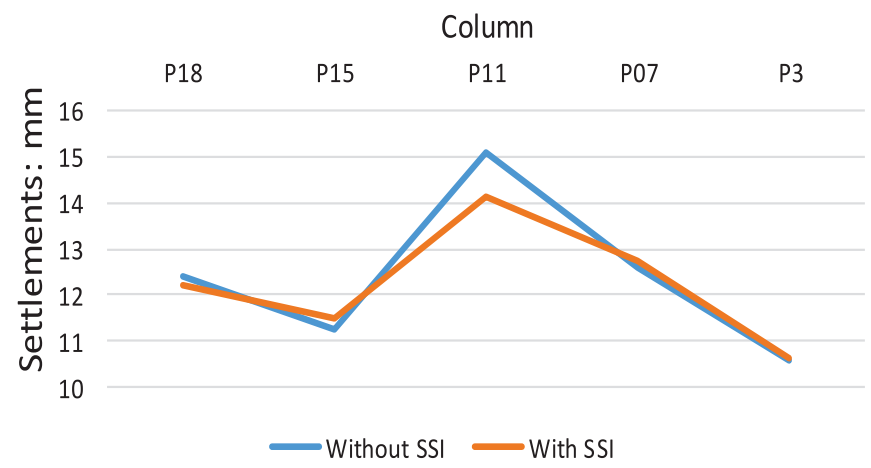

(f)

Figure 6

Settlement curve for the 4-floor building case 


\section{Table 10}

Values of the Gamma-Z instability parameter and its respective variations

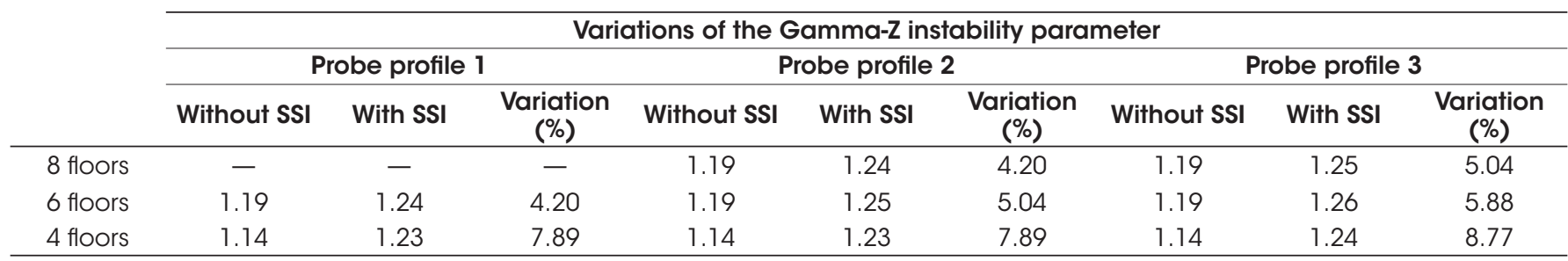

\section{Table 11}

Cost of the infrastructure's structural elements and their respective variations

\begin{tabular}{lcccccc} 
& \multicolumn{5}{c}{ Cost of structural elements (R\$) } \\
\cline { 2 - 7 } & \multicolumn{4}{c}{ Piles } & \multicolumn{3}{c}{ Blocks } \\
\cline { 2 - 7 } & Without SSI & With SSI & $\begin{array}{l}\text { Var. } \\
\text { (\%) }\end{array}$ & Without SSI & With SSI & $\begin{array}{c}\text { Var. } \\
\text { (\%) }\end{array}$ \\
\hline 8 floors - probe profile 2 & 102514.97 & 102514.97 & 0.00 & 71691.34 & 71102.96 & -0.82 \\
8 floors - probe profile 3 & 73551.27 & 73551.27 & 0.00 & 51941.38 & 51333.72 & -1.17 \\
6 floors - probe profile 1 & 98979.81 & 98979.81 & 0.00 & 64739.00 & 64701.91 & -0.06 \\
6 floors - probe profile 2 & 66548.80 & 66548.80 & 0.00 & 43499.83 & 43614.59 & 0.26 \\
6 floors - probe profile 3 & 47540.96 & 47540.96 & 0.00 & 31399.57 & 31387.60 & -0.04 \\
4 floors - probe profile 1 & 60779.13 & 60779.13 & 0.00 & 24963.51 & 25047.32 & 0.34 \\
4 floors - probe profile 2 & 42202.43 & 42202.43 & 0.00 & 17399.81 & 17325.35 & -0.43 \\
4 floors - probe profile 3 & 33255.06 & 33255.06 & 0.00 & 14654.82 & 14584.74 & -0.48 \\
\hline
\end{tabular}

\subsection{Material costs}

Table 11 shows the influence of SSI on the costs of the structura elements of the infrastructure. SSI did not influence the cost of piles, because even with the changes in loads, the stresses stayed below $5 \mathrm{MPa}$. As for the pile caps, no tendency for variation was found, because in the sizing of these elements, the final result depends on the envelopment of the final forces, which varied between favorable and unfavorable, but was relatively small.

Table 11 Cost of the infrastructure's structural elements and their respective variations

Table 12 shows the influence of SSI on the costs of the structural elements of the superstructure. The costs of the slabs and stairs did not change. As for the beams, no tendency for variation was found, because in the sizing of these elements, the final result depends on the envelopment of the final forces, which were sometimes favorable and others unfavorable, but always relatively small. The costs of the columns always increased because the structures always had greater displacements after considering the SSI, causing secondorder forces to increase and, consequently, also the amount of steel. Table 13 shows the costs of global, infrastructure and superstructure costs. The type of soil did not significantly influence the cost of the structure after the analysis with SSI, because when soil resistance was lower, the foundations required piles and pile caps with larger dimensions, compensating with their degree of stiffness. The infrastructure cost was slightly reduced in 6 of the 8 cases under study. The infrastructure of the buildings had their costs reduced after considering the SSI because when the settlements in the

\section{Table 12}

Cost of the superstructure's structural elements and their respective variations

\begin{tabular}{|c|c|c|c|c|c|c|c|c|c|}
\hline & \multicolumn{9}{|c|}{ Cost of structural elements (R\$) } \\
\hline & \multicolumn{4}{|c|}{ Beams } & \multicolumn{2}{|c|}{ Columns } & \multicolumn{3}{|c|}{ Slabs and Stairs } \\
\hline & $\begin{array}{c}\text { Without } \\
\text { SSI }\end{array}$ & With SSI & $\begin{array}{l}\text { Var. } \\
\text { (\%) }\end{array}$ & $\begin{array}{c}\text { Without } \\
\text { SSI }\end{array}$ & With SSI & $\begin{array}{l}\text { Var. } \\
(\%)\end{array}$ & Without SSI & With SSI & $\begin{array}{l}\text { Var. } \\
(\%)\end{array}$ \\
\hline 8 floors - probe profile 2 & 157802.12 & 157388.94 & -0.26 & 69890.93 & 71552.12 & 2.38 & 313360.21 & 313360.21 & 0.00 \\
\hline 8 floors - probe profile 3 & 157802.12 & 158117.48 & 0.20 & 69693.54 & 70929.58 & 1.77 & 313360.21 & 313360.21 & 0.00 \\
\hline 6 floors - probe profile 1 & 120257.41 & 120066.44 & -0.16 & 41157.29 & 41674.71 & 1.26 & 232308.38 & 232308.38 & 0.00 \\
\hline 6 floors - probe profile 2 & 120257.41 & 120201.64 & -0.05 & 41010.70 & 41105.02 & 0.23 & 232308.38 & 232308.38 & 0.00 \\
\hline 6 floors - probe profile 3 & 120257.41 & 120536.27 & 0.23 & 41072.02 & 41592.07 & 1.27 & 232308.38 & 232308.38 & 0.00 \\
\hline 4 floors - probe profile 1 & 85725.14 & 85601.62 & -0.14 & 20839.41 & 21462.25 & 2.99 & 157954.09 & 157954.09 & 0.00 \\
\hline 4 floors - probe profile 2 & 85725.14 & 85701.48 & -0.03 & 20772.54 & 20950.66 & 0.86 & 157954.09 & 157954.09 & 0.00 \\
\hline 4 floors - probe profile 3 & 85725.14 & 85819.74 & 0.11 & 20790.65 & 21037.68 & 1.19 & 157954.09 & 157954.09 & 0.00 \\
\hline
\end{tabular}


Global, infrastructure and superstructure costs and their respective variations

\begin{tabular}{|c|c|c|c|c|c|c|c|c|c|}
\hline & \multicolumn{9}{|c|}{ Global, infrastructure and superstructure costs (R\$) } \\
\hline & \multicolumn{3}{|c|}{ Infrastructure costs } & \multicolumn{3}{|c|}{ Superstructure costs } & \multicolumn{3}{|c|}{ Global costs } \\
\hline & W/O SSI & W/ SSI & $\begin{array}{l}\text { Var. } \\
\text { (\%) }\end{array}$ & W/O SSI & W/ SSI & $\begin{array}{l}\text { Var. } \\
\text { (\%) }\end{array}$ & W/O SSI & W/ SSI & $\begin{array}{l}\text { Var. } \\
\text { (\%) }\end{array}$ \\
\hline 8 floors - probe profile 2 & 174206.31 & 173617.93 & -0.34 & 541053.26 & 542301.26 & 0.23 & 715259.57 & 715919.19 & 0.09 \\
\hline 8 floors - probe profile 3 & 125492.64 & 124884.99 & -0.48 & 540855.86 & 542407.26 & 0.29 & & 667292.25 & 0.14 \\
\hline 6 floors - probe profile 1 & 163718.81 & 163681.72 & -0.02 & 393723.08 & 394049.53 & 0.08 & 557441.88 & 557731.26 & 0.05 \\
\hline 6 floors - probe profile 2 & 110048.63 & 110163.39 & 0.10 & 393576.49 & 393615.04 & 0.01 & 503625.13 & 503778.43 & 0.03 \\
\hline 6 floors - probe profile 3 & 78940.53 & 78928.56 & -0.02 & 393637.81 & 394436.72 & 0.20 & 472578.34 & 473365.28 & 0.17 \\
\hline 4 floors - probe profile 1 & 85742.64 & 85826.44 & 0.10 & 264518.63 & 265017.95 & 0.19 & 350261.27 & 350844.40 & 0.17 \\
\hline 4 floors - probe profile 2 & 59602.24 & 59527.78 & -0.12 & 264451.76 & 264606.23 & 0.06 & 324054.01 & 324134.01 & 0.02 \\
\hline 4 floors - probe profile 3 & 47909.89 & 47839.81 & -0.15 & 264469.87 & 264811.51 & 0.13 & 312379.76 & 312651.32 & 0.09 \\
\hline
\end{tabular}

foundations are considered, their bending moments decreased. Consequently, the superstructure had to become more rigid, absorbing part of the forces that would be transferred to the foundations, which meant their costs increased. As for the superstructure, its costs always increased, even with relatively small variations. The global costs of the buildings under analysis always increased after considering SSI, but the variations were relatively low, with the largest difference being $0.17 \%$. This is because the infrastructure had negative variations and the superstructure had positive variations, which meant some of the values were compensated.

\section{Conclusions}

In this paper, reinforced concrete buildings with drilled piles and pile caps were analyzed, generating 8 case studies with and without considering SSI. The results revealed that:

- Half of the cases did not follow the general trend of reducing loads and settlements for the central columns and increasing loads for those of the periphery, and this trend ceased to exist for buildings with fewer floors;

- The variation of the loads and settlements in the foundations had no significant changes for the 8 models under analysis;

- SSI influenced the Gamma-Z global instability index of all cases and its values were always increased after considering the SSI;

- The cost of the structures was not significantly influenced by the type of soil. Still, in general, the infrastructure costs of the buildings reduced slightly and the global costs had a small increase, with a maximum variation of $0.17 \%$.

When the foundation design is performed coherently for small buildings with deep pile foundations, the SSI effects are not significant and can be considered as included in the intrinsic uncertainties of the superstructure and foundation design process, which are considered in the internal safety coefficients established in the NBR 6118 and NBR 8661 standards.

\section{Acknowledgments}

The authors would like to thank the Community University of the Chapecó Region (UNOCHAPECÓ) for the incentive to perform this work and also TQS Informática Ltda. for making the CAD/TQS software available.

\section{Bibliographical references}

[1] ASSOCIAÇÃO BRASILEIRA DE NORMAS TÉCNICAS. Projeto e Execução de Fundações. - NBR 6122, Rio de Janeiro, 2010.

[2] KAUSEL, E. Early history of soil-structure interaction. Soil Dynamics and Earthquake Engineering. v.30, 2010; p. 822-832.

[3] GUSMÃO, A. D. Estudo da interação solo-estrutura e sua influência em recalques de edificações, Rio de Janeiro, 1990. Dissertação (mestrado) - Universidade Federal do Rio de Janeiro, $165 \mathrm{p}$.

[4] ANTONIAZZI, J. P.; ALVA, G. M. S.; SOARES, J. M. D. Metodologia simplificada para a construção da interação solo-estrutura em edifícios com fundações em sapatas isoladas. Engenharia Estudo e Pesquisa, Santa Maria, v. 10, n. 2. p. 3-14, abr./dez. 2010.

[5] PAVAN, R.C.; COSTELLA, M. F.; GUARNIERI, G. Interação solo-estrutura para sistemas estruturais reticulados sobre fundações raras. Revista IBRACON de Estruturas e Materiais, v. 7, n. 2, 2014; p. 260-285.

[6] LIMA, A. I. C.; SILVA R. J. C.; SILVA FILHO, F. C. Influência dos recalques do radier na edificação. In: Congresso Brasileiro do Concreto CBC, $49^{\circ}$, Bento Gonçalves, 2007, Anais, Rio Grande do Sul, 2007.

[7] ROSA, L. M. P.; DANZIGER, B. R.; CARVALHO, E. M. L. Interação solo-estrutura: análise contemplando fluência e retração do concreto. Revista IBRACON de Estruturas e Materiais, v. 11, n. 3, 2018; p. 564-585.

[8] SAVARIS, G.; HALLAK, P. H.; MAIA, P. C. Influence of foundation settlements in load redistribution on columns in a monitoring construction - case study. Revista IBRACON de Estruturas e Materiais, v. 3, n. 3, 2010; p. 346-356.

[9] MOTA, M. M. C. Interação solo-estrutura em edifícios com fundação profunda: método numérico e resultados observados no campo, São Carlos, 2009, Tese (doutorado) - Universidade de São Paulo, 221 p.

[10] BORGES, A. C. L. Metodologia para avaliação do comportamento estrutural de edifícios altos em concreto armado com consideração da interação solo-estrutura, 
Recife, 2009, Tese (doutorado) - Universidade de São Paulo, $221 \mathrm{p}$.

[11] SCHEPERS, W. Fast 3D FEM-BEM coupling for dynamic soil-structure interaction. In: X International Conference on Strcuctural Dynamics, EURODYN 2017, Berlim, 2017, Anais, Germany, 2017, p. 391-396.

[12] RESTREPO, L. R.; JARAMILLO, J. D.; Identificación de propriedades dinámicas de estrutcturas considerando efectos de interacción suelo-estructura (caso de studio). Revista Internacional de Desastres Naturales, Accidentes e Infraestructura Civil, v. 8, n. 2, 2008; p. 135-150.

[13] PAPADOPOULOS, M.; VAN BEEUMEN, R.; FRANÇOIS, S.; DEGRANDE, G.; LOMBART, G. Computing the modal characteristics of structures considering soil-structure interaction effects. In: X International Conference on Strcuctural Dynamics, EURODYN 2017, Berlim, 2017, Anais, Germany, 2017, p. 391-396.

[14] CARBONARI, S.; DEZI, F.; LEONI, G. Linear soil-structure interaction of coupled wall-frame structures on pile foundations. Soil Dynamics and Earthquake Engineering. v. 31, 2011; p. 1296-1309.

[15] GUSMÃO, A. D. A interface entre a engenharia geotécnica e a estrutural. In: Encontro Nacional de Engenharia e Consultoria Estrutural, $17^{\circ}$, São Paulo, 2014, Anais, São Paulo, 2014.

[16] VENANZI, I.; SALCIARINI, D.; TAMAGNINI, C. The effect of soil-foundation-structure interaction on the wind-induced response of tall buildings. Engineering Structures. v. 79, 2014; p. 117-130.

[17] ROBERTO, L.; SOLA, F. Influencia de la interacción dinámica suelo-estructura en la respuesta dinámica de las estructuras. Construcción y tecnologia en concreto, Mayo, 2013.

[18] IWAMOTO, R. K. Alguns aspectos dos efeitos da interação solo-estrutura em edifícios de múltiplos andares com fundação profunda, São Paulo, 2000, Dissertação (mestrado) - Universidade de São Paulo, $157 \mathrm{p}$.

[19] ANTONIAZZI, J. P. Interação solo estrutura de edifícios com fundações superficiais, Santa Maria 1995, Dissertação (mestrado) - Universidade Federal de Santa Maria, $138 \mathrm{p}$.

[20] MENDES, R. S. Interação solo-estrutura e sua influência na análise estrutural de edifícios em concreto armado. Revista Especialize On-line IPOG, v. 01, Dez. 2015.

[21] JORDÃO, D. R. Estabilidade global de edifícios sobre fundações profundas, considerando a interação estrutura-solo, São Paulo, 2003, Dissertação (mestrado) - Universidade de São Paulo, 132 p.

[22] DELALIBERA, R. G. et al. Estabilidade global de edifícios de concreto armado: análise dos métodos $\mathrm{P}-\Delta \mathrm{e}$ yz considerando a deformabilidade da fundação. In: Congresso Brasileiro Do Concreto, $47^{\circ}$., 2005, Recife. Anais, São Paulo, 2005.

[23] CRESPO, V. A. S. Estudo da sensibilidade de edificações em relação ao solo, Rio de Janeiro, 2004, Dissertação (mestrado) - Universidade Estadual do Norte Fluminense Darcy Ribeiro, Goytacazes, 84 p.
[24] MEDEIROS, D. S. Análise tridimensional da interação solo-estrutura em fundações de concreto armado pelo método dos elementos finitos, Porto Alegre, 2006, Dissertação (mestrado) -Universidade Federal do Rio Grande do Sul, $171 \mathrm{p}$.

[25] AOKI, N.; VELLOSO, D. A. An approximate method to estimate the bearing capacity of piles. Proceedings, 5th, Panamerican Conference on Soil Mechanics and Foundation Engineering, Buenos Aires, Argentina, v. 5, p. 367-376, 1975.

[26] AOKI, N.; LOPES, F. R. Estimating stresses and settlements due to deep foundations by the Theory of Elasticity. Proceedings, 5th Pan American CSMFE, Buenos Aires, v.1, p. 377-386, 1975.

[27] PENNA, Antônio Sérgio Damasco. Elementos estruturais de fundações: Transparências de apoio às aulas de fundações. São Paulo: Programa de Educação Continuada em Engenharia da Escola Politécnica da Universidade de São Paulo, 2004.

[28] TQS INFORMÁTICA LTDA. Manual teórico: sistema de interação solo-estrutura. São Paulo: TQS Informática Ltda, 2015. 